\title{
A pproche sédimentologique pour une résolution optimale des problèmes de pertes de boues de forage
}

\author{
N. Kherfellah ${ }^{1}$, H. A it Salem ${ }^{1}$, K. Bekkour ${ }^{2 *}$ and S. Benhadid ${ }^{3}$

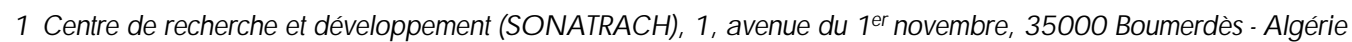 \\ 2 Institut de mécanique des fluides et des solides, UMR ULP-CN RS 7507, 2, rue Boussingault, 67000 Strasbourg - France \\ 3 Université des sciences et de la technologie Houari Boumediene, Institut de physique, Laboratoire de rhéologie, BP 32, El Alia - Algérie \\ e-mail : bekkour@imfs.u-strasbg.fr \\ * Auteur auquel la correspondance doit être adressée
}

Résumé - Malgré les connaissances géologiques accumulées sur plus de 800 puits carottés en continu au cours des quarante dernières années, le forage de la phase 8"' $1 / 2$ du champ pétrolifère de HassiMessaoud (Algérie) reste à ce jour délicat en raison notamment de problèmes de cavage et de pertes de boue. L'objectif de ce travail était d'étudier les problèmes de pertes de boues en utilisant une approche sédimentologique et de localiser les niveaux producteurs sous-jacents du Cambrien. Pour cela, les zones à avancement rapide et lent des forages des séries sédimentaires du Trias ont été repérées afin d'en établir des cartes de répartition. Ainsi, il a été mis en évidence :

- que les zones à avancement rapide correspondent aux secteurs sans pertes de boue (faciès argileux de la plaine d'inondation), alors que les zones à avancement lent correspondent aux secteurs à pertes importantes (faciès gréseux des barres fluviatiles);

- que sous les zones à avancement rapide dans les séries du Trias, se trouvent des réservoirs d'hydrocarbures potentiels.

\footnotetext{
Abstract - A Sedimentological Approach for an Optimal Resolution of Drilling Mud Losses - In spite of the geological knowledge accumulated on more than 800 uninterruptedly cored wells during the forty last years, the drilling of the 8"1/2 diameter phase of Hassi-Messaoud oil field (Algeria) remains delicate because of excavation and drilling mud losses problems. The main objective of this work was to study the problem of drilling mud losses by the use of a sedimentological approach and to localize the producing levels of the subjacent Cambrian. For that purpose, the fast and slow drilling advance zones in the Triassic sedimentary sequences were localized in order to establish distribution maps. Thus, it was highlighted:

- that the fast advance zones correspond to the sectors without mud losses (argillaceous facies of the flood plain), whereas the zones with slow advance correspond to the significant mud losses sectors (sandy facies of the fluviatile bars);

- that under the fast advance zones of the Triassic series, potential hydrocarbon reservoirs are located.
} 


\section{IN TRO DUCTIO N}

Les différents faciès des roches rencontrés au cours d'un forage ont une influence capitale sur sa vitesse d'avancement, notamment lorsque des problèmes de pertes de boues à travers la formation surviennent. Afin de mieux aborder ces questions, il nous est apparu impératif d'étudier la nature du contenant (roche) ainsi que ses effets sur le contenu. On a donc pu constater que les principaux faciès (ou types) de roches rencontrés, tels que les grès (barres sableuses d'origine fluviatile), les silts (limons de débordement) et les argiles (plaines d'inondation) relevaient plutôt d'un problème d'environnement sédimentaire (sédimentologie) : les questions soulevées par les pertes de boues à travers la formation lors d'un forage seraient donc étroitement liées à ces aspects.

Sur l'un des champs pétroliers de la région de HassiMessaoud (Algérie), la phase 2350 à $3400 \mathrm{~m}$ est forée avec un outil de diamètre 8 ' $1 / 2$, avec une pénétration de $4 \mathrm{~m}$ dans le Cambrien (réservoir). Cette phase rencontre des problèmes de cavage et de pertes de boue. Il est donc primordial d'identifier les contraintes dues à ces formations triasiques, de manière à tenter de s'en affranchir au cours de la conception de nouvelles méthodologies. Les difficultés géologiques rencontrées lors du forage de cette phase englobent la tenue des parois du trou, les pressions rencontrées et le contrôle des fluides contenus dans les formations forées. Une étude statistique a été menée sur les puits forés sur le champ de Hassi-Messaoud dans le but de mettre en évidence le degré de virulence des pertes de circulation en phase 8 "' $1 / 2$ des différentes zones du champ.

\section{HISTO RIQ UE DU CHAMP DE HASSI-MESSAOUD}

Le champ de Hassi-Messaoud est un gisement d'hydrocarbures géant qui s'étend sur une superficie d'environ $2000 \mathrm{~km}^{2}$. La couche productrice gréseuse, d'âge cambrien située à une profondeur moyenne de $3400 \mathrm{~m}$, se caractérise par une très grande variabilité de ses propriétés pétrophysiques (porosité et perméabilité). Celles qui sont le plus directement liées à la productivité des puits (porosité, argilosité et perméabilité) sont encore aujourd'hui difficilement prévisibles et ce, malgré les connaissances géologiques accumulées sur plus de 800 puits carottés en continu au cours des quarante dernières années. L'évolution tectonique du Sahara algérien est assez bien connue [1]. Le champ de HassiMessaoud, se localise à la terminaison nord de la dorsale d'Amguid-El Biod, élément structural majeur dépassant $800 \mathrm{~km}$ de longueur, étudié par Heybroek [2]. Ce vaste «trend» a subi à plusieurs reprises des contraintes orogéniques et des déformations notables.

Plusieurs phases orogéniques ont affecté cette région :

- phase néocalédonienne précoce, antétrémadocienne $(\approx 500 \mathrm{Ma})$;
- phase calédonienne majeure $(\approx 400 \mathrm{Ma})$;

- phase hercynienne majeure ( $\approx 225$ à $250 \mathrm{Ma})$;

- phase autrichienne $(\approx 110 \mathrm{Ma})$.

\section{POSITION DU PROBLÈME}

Le but de ce travail est de bien expliciter les problèmes rencontrés lors du forage des puits du champ de HassiMessaoud (fig. 1) en premier lieu et de rechercher, par la suite, les zones à avancement rapide du forage (sans problèmes de pertes de boues) au cours de la traversée des séries triasiques. Ces zones à avancement rapide engendrent un gain de temps et d'argent très appréciable pour le forage du puits. Il s'agira ensuite de s'assurer que sous ces zones, il est possible de rencontrer des réservoirs potentiels d'hydrocarbures dans le Cambrien. De ce fait, nous avons été amenés à :

- localiser et délimiter ces zones à avancement rapide au cours du forage des formations du Trias ;

- voir si leur délimitation correspond à des réservoirs cambriens sous-jacents.

Partant de là, nous avons été amenés à utiliser les schémas paléogéographiques des séries sédimentaires du Cambrien, préalablement établis, afin de vérifier si la plupart des réservoirs cambriens se situent sous ces zones à avancement rapide du forage dans les séries du Trias.

\section{DIFFICULTÉS GÉO LO G IQ UES}

Les différentes formations rencontrées lors du forage de la phase 8 "' $1 / 2$ du champ de Hassi-Messaoud sont décrites comme suit (fig. 1) :

- Lias dolomitique (LD2): cette formation est caractérisée par la présence d'une eau chlorurée calcique et une pression de gisement très élevée qu'il n'est pas possible d'équilibrer par la pression hydrostatique sans provoquer des pertes au niveau du Trias argilo-gréseux (TAG).

- Trias salifère (TS2) : dans cette formation salifère, des argiles de nature plastique d'une épaisseur de $15 \mathrm{~m}$ sont intercalées vers $2900 \mathrm{~m}$. Elles peuvent fluer et refermer le puits dans un temps très court si la densité de boue est insuffisante. La partie salifère, également soumise à cet effet, peut se trouver érodée au cours du forage.

- Trias salifère (TS3) : cette formation est constituée de sel massif avec des traces d'argiles. La traversée en forage 8 ' $1 / 2$ de cette zone à forte concentration en sel provoque un diamètre de trou important.

- Trias argilo-gréseux (TAG) : il est principalement constitué par une succession de passées d'argiles dolomitiques et anhydritiques, voire sableuses et de grès fins, parfois argileux. Le toit de chaque série constituant le Trias argileux gréseux est repéré par un marqueur (G10, G20, etc.). Le repère $\mathrm{G} 35$ est le repère géologique à partir duquel 


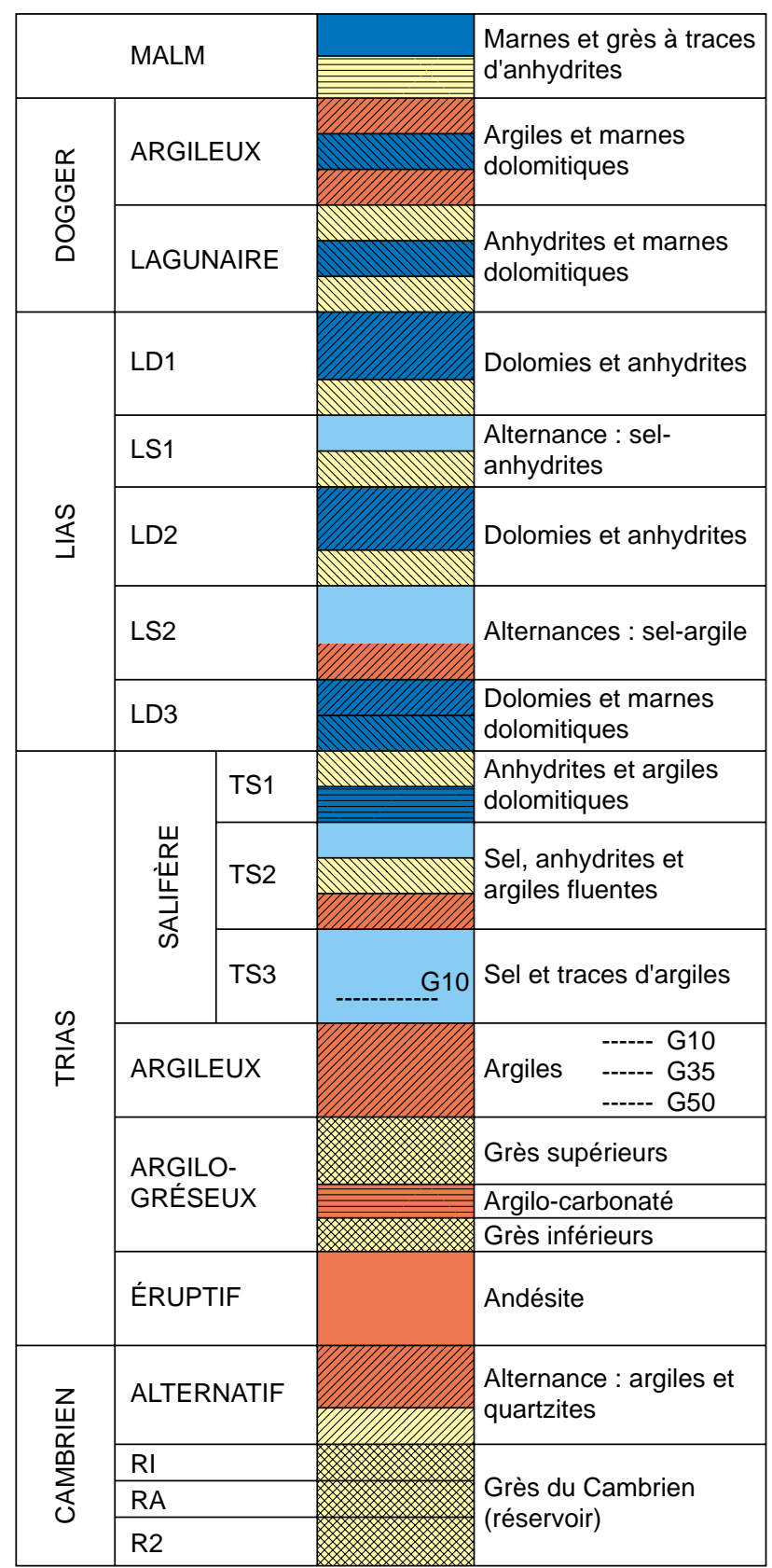

Figure 1

Difficultés géologiques rencontrées lors du forage de la phase 8" $1 / 2$ du champ de Hassi-Messaoud.

Geological difficulties encountered during the drilling of the 8' '1/2 phase of Hassi-Messaoud field.

disparaissent totalement les formations salifères et apparaissent les bancs d'argiles silteuses très indurées, susceptibles de craquer sous la pression hydrostatique de la boue à forte densité nécessaire pour la tenue des eaux chlorurées calciques du $L D 2$ et des argiles fluentes (argiles plastiques du TS2) à taux d'hydratation anormalement élevé traversées depuis le Lias salifère $L S 2$.
Il est à noter que la phase 8 "' $1 / 2$ de la région de HassiMessaoud est la plus délicate à réaliser en raison de la configuration géologique précédemment décrite et des difficultés y découlant rencontrées lors du forage. Celles-ci peuvent être sommairement résumées comme suit :

- diffusion des eaux chlorurées calciques du Lias dolomitique $(L D 2)$. Ce problème dépend en grande partie de la durée de réalisation de la phase ;

- fluage des argiles du Trias salifère (TS2) nécessitant une densité élevée du fluide de forage (2 à 2,20);

- problèmes de pertes de circulation dans la base du Trias argileux et dans le Trias argilo-gréseux, notamment si la densité de la boue est supérieure à 1,98 .

\section{4 ÉTUDE STATISTIQ UE DES PUITS À PERTES}

Les pertes de circulation pendant le forage se produisent entre le repère géologique $G 35$ (disparition totale du sel) et la base du Trias argilo-gréseux (fig. 1). D'après une sélection de puits forés dans la région de Hassi-Messaoud, il a été montré que, dans tous les secteurs, chacun des puits nécessitait au moins un bouchon de ciment afin de colmater les pertes de boues partielles à travers le TAG. Pour le cas des pertes totales, il est impératif de poser le tubage 7" au niveau du $G 35$ afin d'éviter l'abandon du puits. La figure 2 représente la localisation de ces puits avec le nombre de bouchons de ciment posés et le tableau 1 récapitule pour chaque secteur le nombre de puits forés, la position du tubage et le nombre moyen de bouchons de ciment utilisés pour chaque puits.

Au niveau du champ de Hassi-Messaoud, le nombre de bouchons de ciment est de trois. Les secteurs les plus virulents sont: ONM, OMP et OML.

\section{TABLEAU 1}

Principales données de forage (nombre de puits, tubages, bouchons de ciment) par secteur du champ de Hassi-Messaoud

Main drilling informations (number of drilled wells, casing, cement plugs) by sector of Hassi-Messaoud field

\begin{tabular}{c|c|c|c|c}
\hline Secteur & $\begin{array}{c}\text { Nombre total } \\
\text { de puits forés }\end{array}$ & $\begin{array}{c}\text { Nombre de puits } \\
\text { dont le tubage 7’, } \\
\text { est posé au G35 }\end{array}$ & $\begin{array}{c}\text { Taux } \\
(\%)\end{array}$ & $\begin{array}{c}\text { Nombre moyen } \\
\text { de bouchons de } \\
\text { ciment par puits }\end{array}$ \\
\hline OMJ & 11 & 2 & 18 & 3.4 \\
OMK & 5 & 1 & 20 & 2.8 \\
OML & 38 & 15 & 39 & 3.7 \\
OMM & 35 & 7 & 20 & 1.5 \\
OMN & 39 & 1 & 3 & 2.7 \\
OMO & 21 & 1 & 5 & 2.7 \\
OMP & 50 & 29 & 58 & 4.3 \\
ONI & 11 & 3 & 27 & 3.5 \\
ONM & 40 & 20 & 50 & 6.2 \\
MD & 36 & 1 & 3 & 2.1 \\
HMD & 286 & 8 & 28 & 3.1 \\
\hline
\end{tabular}




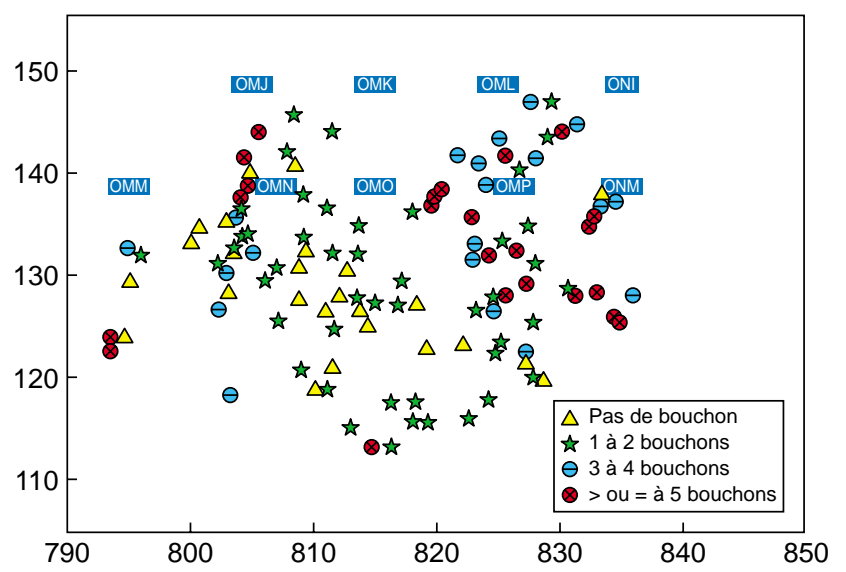

Figure 2

Répartition des puits forés au niveau de la région de HassiMessaoud (1992-1998).

Repartition of drilled wells in Hassi-Messaoud area (19921998).

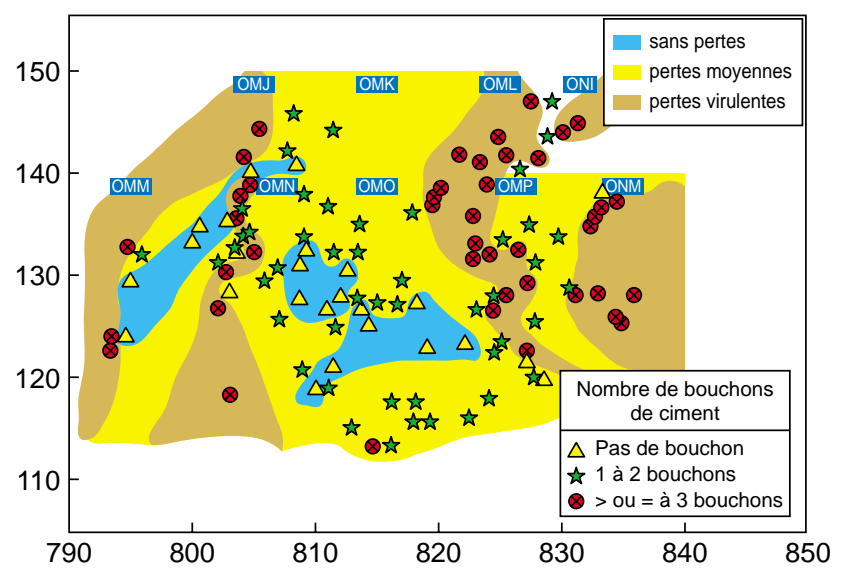

Figure 3

Détermination des chenaux fluviatiles à méandres.

Determination of meander-fluviatile channels.

\section{DÉTERMINATION DES CHEN AUX FLUVIA TILES À MEANDRES DU TAG}

Nous avons remarqué que [3] :

- les niveaux à pertes importantes de boue correspondent à un faciès gréseux (barres fluviatiles sableuses méandriformes mises en évidence par les géologues pétroliers);

- les niveaux sans pertes de boues correspondent à un faciès argileux (plaine d'inondation);

- les niveaux à pertes moyennes en boue correspondent à un faciès silteux (limons de débordement).

À partir de ces observations, une carte délimitant les zones à pertes importantes de boue et les zones sans pertes de boue a été établie (fig. 3). Elle montre que les zones à pertes importantes présentent des zones lenticulaires analogues aux barres sableuses fluviatiles. Ainsi donc, en exploitant les données de forage, nous avons dessiné une carte paléogéographique qui permet de reconstituer, d'une manière globale, un réseau fluviatile où se côtoient les trois faciès principaux suscités et ainsi, de repérer aisément les zones à avancement rapide du forage dans le Trias.

Afin de délimiter les barres sableuses et les plaines d'inondations au niveau de la zone à pertes du Trias argilo-gréseux, nous avons considéré, sur la carte, trois cas de puits :

- à pertes virulentes (en marron) ;

- à pertes moyennes (en jaune) ;

- sans pertes (en bleu).

Nous avons constaté la présence de deux plaines d'inondations :

- La première, à lithologie silteuse ou argilo-silteuse, orientée selon un axe nord-sud, est située dans la partie centrale du champ de Hassi-Messaoud (en jaune sur la carte). On y observe un avancement assez rapide du forage et des pertes de boues moyennes. Elle comporte des zones bien individualisées (en bleu), très argileuses et de formes grossièrement circulaires, au sein desquelles les pertes de boues sont absentes et l'avancement du forage très rapide.

- La seconde, moins étendue, orientée elle aussi selon un axe nord-sud, se situe dans la partie est du champ.

Ces deux plaines d'inondations sont séparées par des chenaux importants, à dominante gréseuse (colorés en marron sur la carte) et orientés selon un axe nord-sud.

\section{LO CALISATION DES SÉQ UEN CES PRO DUCTRICES (PA LÉOG ÉO G RAPHIE)}

Après avoir déterminé les zones à avancement rapide du forage, il est intéressant de localiser les niveaux producteurs sous-jacents du Cambrien. Pour cela, deux méthodes d'analyse des faciès ont été utilisées :

- la première consiste à identifier l'allure de la réponse diagraphique des roches ;

- la seconde, plus précise, consiste à étudier la répartition des lithofaciès et à relever les structures sédimentaires à partir des carottes.

L'analyse des lithofaciès sur des profils verticaux a paru insuffisante, elle doit être complétée par une analyse des éléments architecturaux tels que les chenaux, les barres et formes de couches, les dépôts par accrétion latérale, les dépôts de coulées gravitaires, les dépôts de progragation vers l'aval, les corps sableux tabulaires à laminations et les limons de débordement. 
Des schémas paléogéographiques ont été élaborés par les géologues grâce à l'analyse séquentielle pour délimiter des séquences et des paraséquences [4, 5, 6 ], à savoir :

- une séquence peut être considérée comme une succession relativement conforme de couches génétiquement liées, limitée à sa base et à son sommet par des discordances et par des surfaces corrélables ;

- une paraséquence est une succession relativement conforme de couches ou d'ensembles de couches génétiquement liées, limitée par des surfaces d'inondations marines et par des surfaces corrélables.

Les corrélations entre les sondages plus ou moins éloignés ont été élaborées grâce à l'utilisation des séquences et des paraséquences. À l'aide de ces dernières, des cartes paléogéographiques, mettant en évidence différents lithofaciès, ont pu être réalisées. Cette façon de procéder a permis de mettre en évidence la géométrie et l'orientation des corps sableux qui présenteraient les meilleures propriétés de réservoirs au sein des séries cambriennes.

À titre d'exemple, nous avons étudié les puits MD 50, MD 110, MD 13 et MD 147 situés dans le secteur de coordonnées $[Y \in 120-130]$ et $[X \in 810-820]$. Après avoir superposé les deux cartes paléogéographiques du Trias et du Cambrien, nous avons pu constater que ces quatre puits, situés sous les zones à avancement rapide du forage pendant la traversée du Trias (zone sans pertes de boue), sont producteurs au niveau des paraséquences suivantes :

- paraséquences G, F, E, D, C, B et A, pour le sondage MD 50;

- paraséquence A, pour le sondage MD 110 ;

- paraséquences E, D, C, et A, pour le sondage MD 13;

- paraséquences D, C et A pour le sondage MD 147.

\section{CONCLUSION}

Cette étude avait pour objectif d'apporter des solutions aux problèmes de pertes de boues dans le champ pétrolifère de Hassi-Messaoud (Algérie) en utilisant une approche sédimentologique. Pour cela, nous avons repéré les zones à avancement rapide et lent des forages au cours de la traversée des séries sédimentaires du Trias afin d'en établir les cartes de répartition. Cette opération nous a permis de remarquer que :

- les zones à avancement rapide des forages correspondent aux secteurs sans pertes de boue, c'est-à-dire au faciès argileux de la plaine d'inondation;
- les zones à avancement peu rapide correspondent aux secteurs à pertes importantes en boue, c'est-à-dire au faciès gréseux des barres fluviatiles ;

- les données de forage (avancements au cours du forage, pertes de boues, etc.) ont été combinées aux données de sédimentologie afin de reconstituer une carte des différents niveaux de pertes en boue, reflétant ainsi les faciès traversés et permettant alors de reconstituer globalement la paléogéographie du Trias ;

- la superposition des deux cartes paléogéographiques relatives au Trias et au Cambrien a permis de constater que sous et à l'aplomb des zones à avancement rapide dans les séries du Trias, se trouvent des réservoirs potentiels en hydrocarbures.

Cette façon de procéder, rassemblant les données de la sédimentologie (étude des structures et séquences sédimentaires, répartition des lithofaciès, diagraphies, orientation et géométrie des corps sableux) et celles des études de réservoirs (porosités, perméabilités, données de forage), est économiquement très rentable, puisqu'elle permet $d$ 'atteindre des réservoirs cambriens en un temps très court.

\section{RÉFÉREN CES}

1 Poulet, J. (1967) Étude géochimique des gisements de la région de Hassi-Messaoud - Données géologiques. Rapport IFP $\mathrm{n}^{\circ} 15155$.

2 Heybroek, P. (1963) Note on the Structural Development of the El Biod High and Amguid Spar (Central Algerian Sahara), Revue de l'Institut français du pétrole, $\mathbf{1 8}, 10$.

3 Aït Salem, H. (1992) Le Trias détritique de l'oued Mya (Sahara algérien) - Sédimentation estuarienne - Diagenèse et porogenèse - Potentialités pétrolières. Docum. Lab. Geol. Lyon, 120, 171.

4 Van Wagoner, J.C. (1985) Reservoir Facies Distribution as Controlled by Sea-Level Change. Abstracts, Society of Economic Paleontologists and Mineralogists Midyear Meeting, Golden, Colorado, 91-92.

5 Posamentier, H.W., Jervey, M.T. and Vail, P.R. (1988) Eustatic Control on Clastic Deposition I-Conceptual Framework. In Sea Level Changes: An integrated Approach, Spec. Publ. Soc. Econ. Paleon. Mineral, Eds Wilgus. Tulsa, 42, 109-124.

6 Vail P.R. et al. (1977) Seismic Stratigraphy and Global Changes of Sea Level. In Seismic Stratigraphy. Application to Hydrocarbon Exploration, Amer. Ass. Petr. Geologists Mem., 26, $49-212$.

Manuscrit final reçu en juillet 2002 